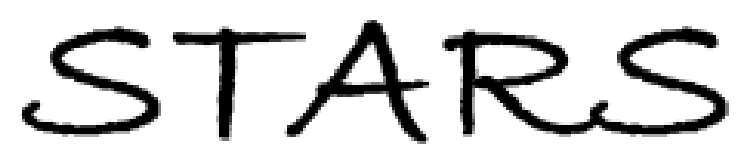

University of Central Florida

STARS

$1-1-2004$

\title{
White-light continuum Z-scan technique for nonlinear materials characterization
}

\author{
Mihaela Balu \\ University of Central Florida \\ Joel Hales \\ University of Central Florida \\ David J. Hagan \\ University of Central Florida \\ Eric W. Van Stryland \\ University of Central Florida
}

Find similar works at: https://stars.library.ucf.edu/facultybib2000

University of Central Florida Libraries http://library.ucf.edu

This Article is brought to you for free and open access by the Faculty Bibliography at STARS. It has been accepted for inclusion in Faculty Bibliography 2000 s by an authorized administrator of STARS. For more information, please contact STARS@ucf.edu.

\section{Recommended Citation}

Balu, Mihaela; Hales, Joel; Hagan, David J.; and Van Stryland, Eric W., "White-light continuum Z-scan technique for nonlinear materials characterization" (2004). Faculty Bibliography 2000s. 4196.

https://stars.library.ucf.edu/facultybib2000/4196

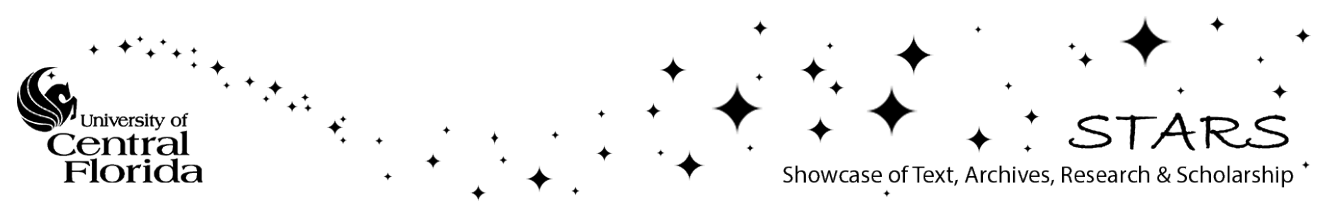




\title{
White-light continuum Z-scan technique for nonlinear materials characterization
}

\author{
Mihaela Balu, Joel Hales, David J. Hagan*, Eric W. Van Stryland* \\ College of Optics \& Photonics: CREOL \& FPCE \\ University of Central Florida \\ 4000 Central Florida Blvd., Orlando, FL 32816-2700 \\ *also with the Department of Physics, University of Central Florida \\ ewvs@creol.ucf.edu
}

\begin{abstract}
We present a technique for rapid characterization of degenerate nonlinear absorption and refraction spectra using a femtosecond white-light continuum (WLC) pulse to perform Z-scans. The spectral components of the WLC source are temporally and spatially dispersed to minimize nondegenerate two-photon absorption (2PA) processes. We demonstrate the validity of the method by measuring the 2PA spectrum of a wellcharacterized semiconductor, $\mathrm{ZnSe}$.
\end{abstract}

(C) 2004 Optical Society of America

OCIS codes: (190.0190) Nonlinear Optics, Materials; (300.0300) Spectroscopy, Nonlinear

\section{References}

1. P.T.C So, C.Y.Dong, B.R.Masters and K.M.Berland, "Two-photon excitation fluorescence microscopy," Annual Review of Biomedical Engineering 2, 399 (2000)

2. K.D. Belfield, X.Ren, D.J.Hagan, E.W. Van Stryland, V.Dubikovsky and E.J.Miesak, "Microfabrication via two-photon photoinitiated polymerization," Abstr. Papers Amer. Chem. Soc. 218, (2001)

3. L.W.Tutt and T.F.Boggess, "A review of optical limiting mechanisms and devices using organics, fullerenes, semiconductors and other materials, " Prog. Quamtum Electron 17, 299 (1993)

4. H.M Gibbs, Optical Bistability: Controlling Light with Light (Academic Press, London, 1985)

5. M.Sheik-Bahae, A.A.Said, T.H.Wei, D.J.Hagan and E.W. Van Stryland, "Sensitive measurement of optical nonlinearities using a single beam," IEEE J.Quantum Electron. 26, 760 (1990)

6. R. Negres, J. Hales, A. Kobyakov, D. J. Hagan and E. W. Van Stryland, "Experiment and analysis of twophoton absorption spectroscopy using a white-light continuum probe," IEEE J.Quantum Electron. 38, 1205 (2002)

7. G.S.He, T.C Lin, P.N. Prasad, R.Kannan, R.A. Vaia and L.S. Tan, " New technique for degenerate twophoton absorption spectral measurements using femtosecond continuum generation,” Opt. Express 10, 566 (2002)

8. E.W.Van Sryland, M.A. Woodall, H.Vanherzeele and M.J.Soileau, "Energy band-gap dependence of twophoton absorption," Opt. Lett. 10, 490 (1985)

9. A.K.Dharmadhikari, F.A.Rajgara, N.C.S.Reddy, A.S.Sandhu and D.Mathur, "Highly Efficient White Light Generation from Barium Fluoride,” Opt. Express 12, 695 (2004)

10. A. Brodeur, F.A. Ilkov, S.L. Chin, "Beam filamentation and the white light continuum divergence," Opt. Commun. 129, 193 (1996)

11. S.Yamaguchi and H. Hamaguchi, "Convenient method of measuring the chirp structure of femtosecond white-light continuum pulses," Appl. Spectrosc. 49, 1513 (1995)

12. A.A.Said, M.Sheik-Bahae, D.J. Hagan, T.H.Wei, J.Wang, J.Young and E.W.Van Stryland, "Determination of bound-electronic and free-carrier nonlinearities in ZnSe, GaAs, CdTe and ZnTe," J. Opt. Soc. Am. B 9, 405 (1992)

13. M.Sheik-Bahae, A.A.Said and E.W. Van Stryland, "High-sensitivity, single-beam $\mathrm{n}_{2}$ measurements," Opt. Lett. 14, 955 (1989)

14. M.Balu, J.Hales, D.J.Hagan and E.W.Van Stryland, "White-light continuum z-scan technique for nonlinear material characterization," CLEO Conference, San Francisco, May 16-21, 2004 


\section{Introduction}

Recently there has been intense interest in applications which exploit two-photon absorption (2PA) such as three-dimensional fluorescence imaging [1] and microfabrication [2] as well as optical limiting [3], while the optically-induced change in refractive index is fundamental to all-optical switching [4]. The quest for materials exhibiting high nonlinear refraction (NLR) and absorption (NLA) requires methods for rapid characterization over a broad spectral range.

The well-established single wavelength Z-scan [5] is a popular technique because of its simplicity, sensitivity, accuracy and ease of separation of NLA and NLR. It is a rapid method to determine nonlinear coefficients for a particular wavelength, but obtaining a broad spectral dependence of the nonlinearity requires wavelength tuning and realignment which are often time consuming processes.

In this article we demonstrate that we can overcome this shortcoming by replacing the single wavelength source conventionally used in the Z-scan technique with an intense broadband white-light continuum (WLC) source. Previously, WLC sources have been primarily used as weak probes of nonlinear absorption induced by a separate, strong, singlewavelength excitation beam, thus yielding the nondegenerate nonlinearities [6]. However, knowledge of a material's degenerate nonlinearities is often needed for most of the aforementioned applications.

He et. al. recently demonstrated that, using only an intense WLC pulse, degenerate 2PA may be rapidly determined over a broad spectral range [7]. This was accomplished by monitoring the nonlinear transmission of the WLC through a chromophore thus producing a relative spectral curve which is subsequently converted to an absolute scale with an additional nonlinear transmission experiment performed using a separate single wavelength source. While this method is relatively rapid, it requires significant transmittance losses and requires a separate calibration.[5]

Here, we employ the Z-scan technique by using an intense WLC source in place of the single wavelength source conventionally used. This method provides an absolute measurement of the degenerate nonlinearities of the sample under investigation and adds the sensitivity of the normal Z-scan technique. To confirm its validity we test this new procedure on the well-characterized semiconductor, $\mathrm{ZnSe}[5,6,8]$. We demonstrate the potential of this technique for NLA measurements and propose its extension to NLR measurements. The technique we propose, the WLC Z-scan, has the advantage of yielding sensitive, accurate, broadband, simultaneous and rapid measurements of both the degenerate 2PA spectrum and the associated dispersion of the corresponding nonlinear refraction.

\section{Experiment: method and results}

As our pump source, we employed a regeneratively-amplified femtosecond laser producing $\sim 1 \mathrm{~mJ}, \sim 150$ fs pulses at a $1 \mathrm{kHz}$ repetition rate operating at $775 \mathrm{~nm}$. Using a $150 \mathrm{~mm}$ focal length lens, a small portion $(10 \mu \mathrm{J})$ of this beam was focused into a $3 \mathrm{~cm}$ long cell filled with distilled water. This generated a WLC with sufficient energy at many wavelengths suitable for performing Z-scans (note: recently using a $10 \mathrm{~cm}$ thick crystal of $\mathrm{BaF}_{2}$, significantly more WLC energy has been produced [9] ). At the high-powers, white light generation is expected to occur in small-scale filaments [10]. We checked for this experimentally by translating a CCD camera through the focal point where we performed the Z-scan experiment. We found the white light to be in a single beam for the power levels at which we performed the Z-scans. However, at much higher powers we did notice that the white light was composed of multiple beams. Narrow band filters (NBF) of $\sim 10 \mathrm{~nm}$ full-width at half maximum (FWHM) bandwidth were used to characterize the energy in the continuum as a function of wavelength. The energies measured were in the range of $10 \mathrm{pJ} / \mathrm{nm}-25 \mathrm{~nJ} / \mathrm{nm}$ over the range $650 \mathrm{~nm}-900$ $\mathrm{nm}$. Reflection coated optics were used to flatten the spectrum of the WLC. 
To determine the spatial characteristics of the WLC, a knife-edge technique was used to measure the beam waist of different wavelengths in the continuum. These wavelengths were selected by using NBF in the WLC path. To ascertain temporal features of the WLC, we performed an Optical Kerr Gate measurement (OKG) [11] using a thin cell filled with $\mathrm{CS}_{2}$ (0.6 $\mathrm{mm}$ thick) as the Kerr medium. This measurement gave us the temporal separation between different wavelengths of the order of $60 \mathrm{fs}$ per $10 \mathrm{~nm}$. By extracting the temporal cross-correlation traces we were able to determine the pulsewidth for any fixed wavelength in the continuum spectrum for $\sim 10 \mathrm{~nm}$ spectral bandwidths. The value determined was $\sim 180 \mathrm{fs}$ FWHM in the range $670 \mathrm{~nm}-800 \mathrm{~nm}$. With knowledge of the energy, beam waist, and pulse duration for each spectral component of the WLC source, we were able to fit the Z-scan transmission curve [5] corresponding to each wavelength.

In order to confirm the validity of the WLC Z-scan technique we chose to measure the 2PA spectrum of $\mathrm{ZnSe}$. ZnSe was selected since its 2PA spectrum has been well documented in the literature $[5,8]$. Its measured nonlinear refraction spectrum will be reported in a future publication. The thickness of the sample was $0.5 \mathrm{~mm}$ which guaranteed that the thin sample approximation was valid [5]. In order to make the distinction between degenerate and nondegenerate nonlinearities, the 2PA spectrum of $\mathrm{ZnSe}$ was measured at specific wavelengths in the presence of and in the absence of the entire broadband WLC source.

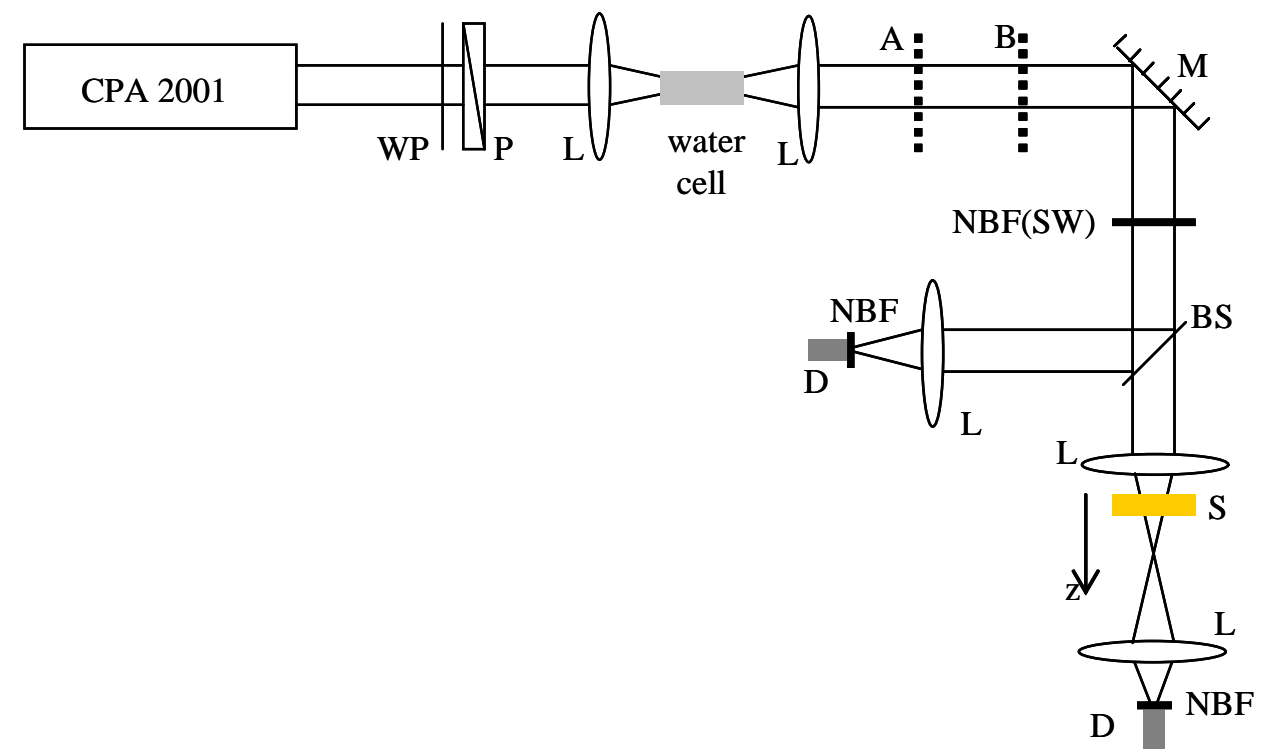

Fig. 1. WLC Z-scan experimental setup. CPA-2001-femtosecond source; WP-waveplate; Ppolarizer; L-lens; GP-glass prism; M-mirror; NBF-narrow band filter; BS-beamsplitter; Ssample; $\mathrm{D}_{1}, \mathrm{D}_{2}$-detectors. $\mathrm{A}$ and $\mathrm{B}$ denote the positions of additional elements inserted in the set-up. $\mathrm{NBF}(\mathrm{SW})$ is used only in the single wavelength configuration discussed in the text with the other two NBF's removed.

The experimental setup is depicted in Fig. 1. In the first experiment, we used the WLC without any external dispersive elements in the beam. As in a normal Z-scan, the WLC beam is focused and the transmittance of the nonlinear sample is monitored as it is scanned along the beam axis through the focal region while the energy in the WLC is held constant. The WLC was stable, but to avoid any possible fluctuations we used an "energy window" on the reference detector (i.e. accepted data only if the reference energy was within a specified energy range). However, the difference between data taken with and without this window was 
not significant. We took data averaging over 500 shots. Since we are reporting on measurements of NLA only, the transmission detector collects the entire beam area. The transmittance of the WLC through the sample was monitored at different wavelengths by placing a narrow band filter (NBF) at a particular wavelength in front of each detector. The Zscan was then repeated for additional wavelengths in order to build up the 2PA spectrum. Figure 2(a) shows a Z-scan trace and the corresponding value of the 2PA coefficient $(\beta)[5,8]$ obtained for one of the wavelengths, $650 \mathrm{~nm}$. The fitted curve uses the focused beam size measured by the knife-edge scans. The curve appears narrower than the experimental data and the value of $\beta$ obtained is much larger than extrapolated from measurements at other wavelengths [8]. The most likely reason for these discrepancies is that since the entire WLC is passing through the sample, other wavelengths can affect the transmission of the detected wavelength through nondegenerate processes such as nondegenerate $2 \mathrm{PA}$, resulting in measurement of an erroneously high 2PA coefficient. The large width of the Z-scan curve may again be due to the nondegenerate $2 \mathrm{PA}$ along with chromatic aberration which causes different wavelengths to focus in different planes [12]. We also found considerable variation in focal spot size at different wavelengths from the knife-edge scans. To verify that nondegenerate $2 \mathrm{PA}$ was occurring, we compared the values of the 2PA coefficients extracted from Z-scan curves in the WLC configuration with those obtained from single-wavelength Zscan experiments performed at the same wavelengths. For the single wavelength Z-scan experiments a narrow band filter was placed in the WLC path, before the sample, creating a quasi-monochromatic beam. Again, by changing the narrow-band filters and repeating the $\mathrm{Z}$ scan, a spectrum of $2 \mathrm{PA}$ could be obtained. Because a NBF placed in the WLC path before the sample infers a single wavelength (SW) experiment, it was denoted $\mathrm{NBF}(\mathrm{SW})$ in Fig. 1. This experimental arrangement will be referred to as "SW configuration" while the one for which the NBF are placed in front of the detectors is referred to as the "WLC configuration". Figure 2(b) shows the experimental data and the corresponding $\beta$ for the SW Z-scan configuration using the same wavelength as in Fig. 2(a). This data gives a better fit and a 2PA coefficient in close agreement with values previously reported in the literature [8].

a)

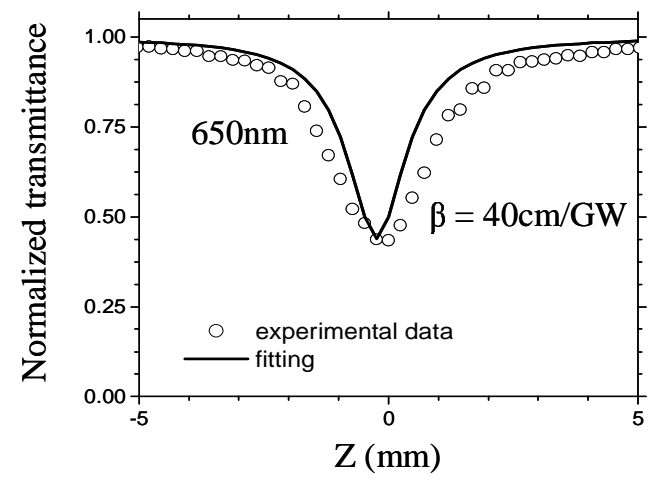

Fig. 2. Normalized Z-scan transmittances of $\mathrm{ZnSe}$ measured at $650 \mathrm{~nm}$ for a) the WLC configuration and b) the SW configuration. The solid lines represent fittings used to extract $\beta$ values.

From the comparison of Fig. 2(a) and 2(b), we attribute the discrepancy between the two values of $\beta$ to the nondegenerate 2PA processes induced in the WLC configuration due to the spatial and partial temporal overlapping between adjacent wavelengths. The temporal overlap 
is only partial because of the temporal dispersion of frequency components of the WLC caused by the group velocity dispersion (GVD) induced by the generating medium, here water. To avoid these nondegenerate nonlinearities, wavelengths need to be either temporally or spatially dispersed prior to entering the sample.

To temporally disperse the WLC, we placed a thick piece of $\mathrm{ZnSe}(7 \mathrm{~mm})$ in the WLC at the position labeled A in Fig. 1. ZnSe possesses large GVD in the visible which imposes a large temporal dispersion on the WLC. The GVD also increases the pulsewidth but for our $180 \mathrm{fs}$ input pulsewidths, the effect of pulsewidth broadening is less significant than the temporal separation of the wavelengths. However, we had to account for this pulse broadening, which resulted in $211 \mathrm{fs}$ at $800 \mathrm{~nm}$ to $247 \mathrm{fs}$ at $670 \mathrm{~nm}$, in order to calculate the irradiance needed to fit the 2PA coefficient to the data at each wavelength. Figure 3(a) and 3(b) show Z-scan traces taken in both WLC and SW Z-scan configurations respectively, at a wavelength of $670 \mathrm{~nm}$. The discrepancy between the value of the 2PA coefficient extracted from the WLC experiment and the one obtained in the SW Z-scan experiment is less severe than in the first experiment but it is still significant, implying that the temporal dispersion imposed by the 7-mm ZnSe was insufficient.

a)

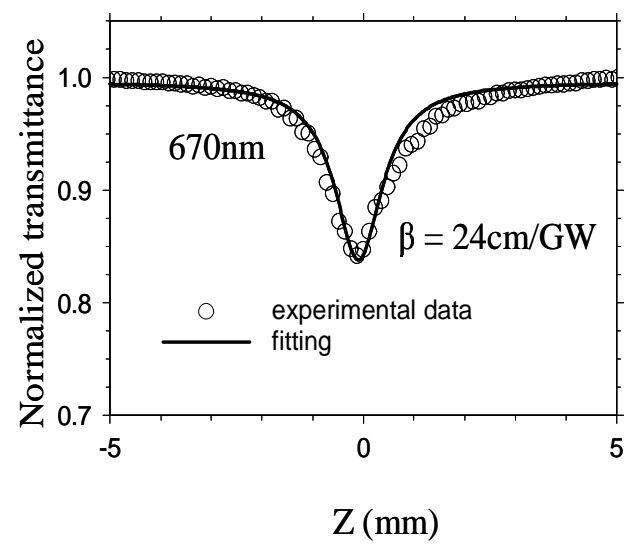

b)

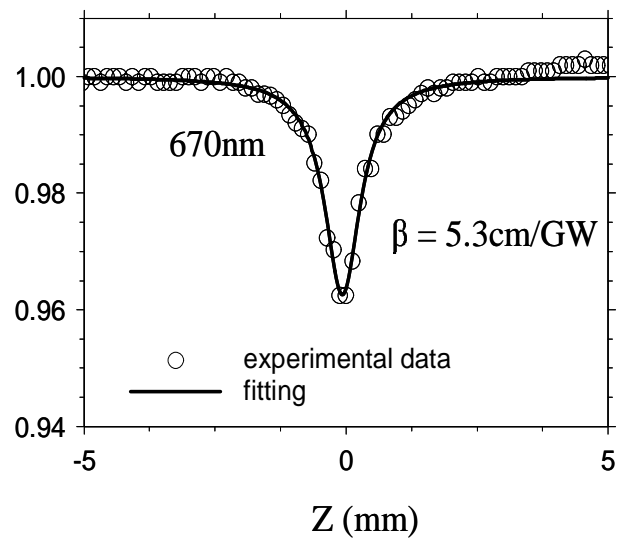

Fig. 3. Normalized Z-scan transmittance of ZnSe measured at $670 \mathrm{~nm}$ with the WLC beam temporally dispersed. The solid lines represent fittings used to extract $\beta$ values a) WLC configuration, b) SW configuration.

In the next experiment the wavelengths in the WLC were spatially dispersed by a glass prism [7] at position B. We repeated the same experiment for different wavelengths and in both configurations. This time, the values of the 2PA coefficients obtained from the fittings for the two configurations were even closer than before (within a factor of 3), suggesting that the spatial separation is more effective than its temporal counterpart in this particular configuration but nonetheless the separation is still insufficient.

Finally, we combined both the $\mathrm{ZnSe}$ for temporal dispersion and the glass prism for spatial dispersion in one experiment. Figure 4(a) and 4(b) show Z-scan curves taken at 670nm in the WLC and the SW configurations. 
a)

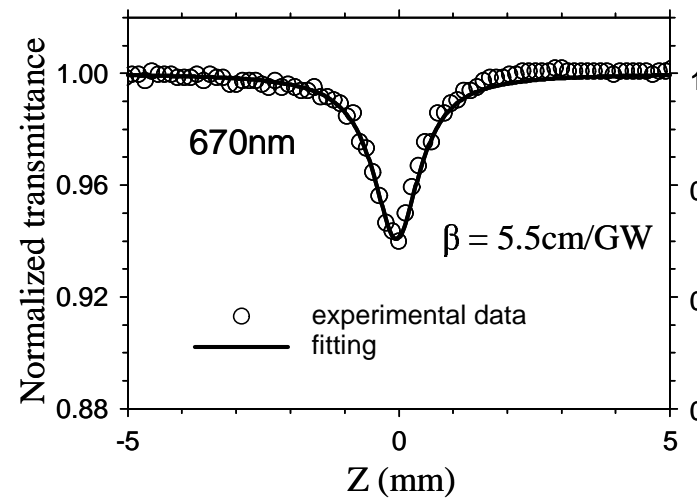

b)

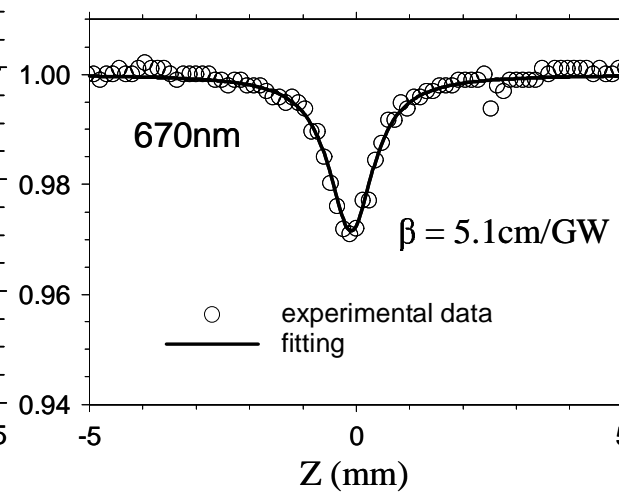

Fig. 4. Normalized Z-scan transmittance of ZnSe measured at $670 \mathrm{~nm}$ with the WLC beam temporally and spatially dispersed. The solid lines represent fittings used to extract $\beta$ values a) WLC configuration, b) SW configuration.

We now obtain good agreement between the values of $\beta$ in the two experiments. We interpret this to show that the wavelength separation was sufficient to make the contributions from nondegenerate 2PA negligible such that the WLC Z-scan truly measures the degenerate 2PA spectrum. Figure 5 shows Z-scan curves taken for this experimental arrangement, in the WLC configuration for different wavelengths. The transmission curves correspond to five different wavelengths sampled by the narrow band filters placed directly before the detectors. The solid lines represent fittings [5] of the experimental curves used to extract values of $\beta$.

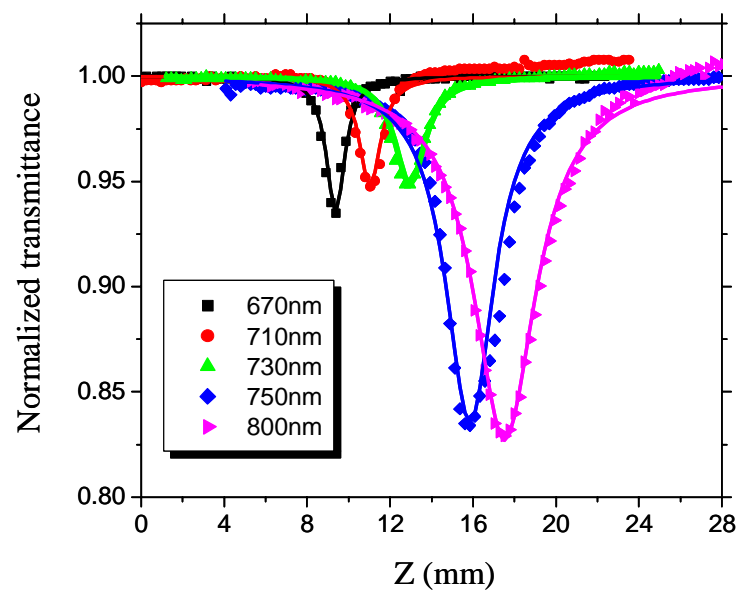

Fig. 5. Normalized Z-scan transmittance of ZnSe measured at different wavelengths with the WLC beam temporally and spatially dispersed. The solid lines represent fittings used to extract $\beta$ values. Z-scan traces are shifted from their original positions for an easier view. 
These values show reasonable agreement with the values obtained from theory [8] as presented in Fig. 6, further confirming the validity of our method for obtaining degenerate nonlinearities.

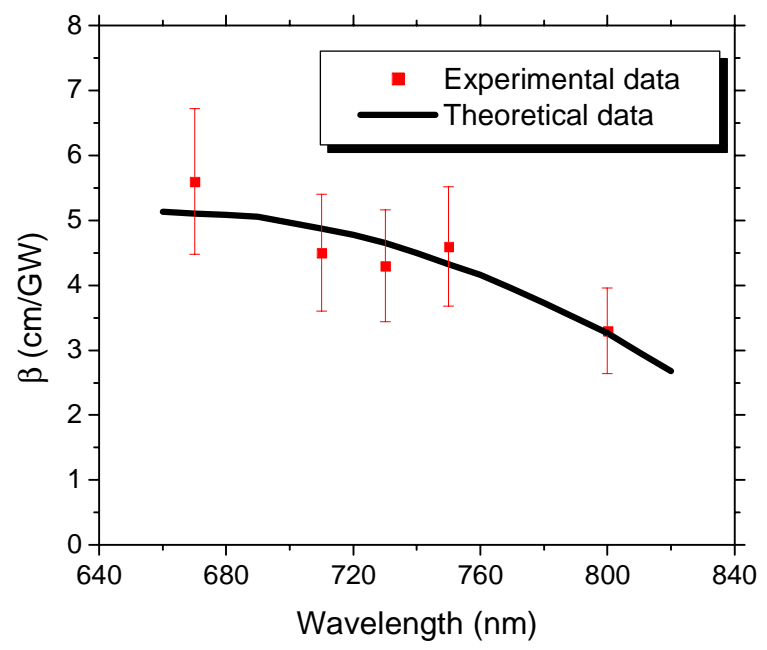

Fig. 6. 2PA coefficient, $\beta$, values obtained from theory and from the experimental data fittings.

\section{Conclusion and future work}

We have demonstrated a sensitive and broadband characterization technique for nonlinear absorption while achieving good agreement between experimental results and theoretical calculations for the 2PA spectra of $\mathrm{ZnSe}$, a well characterized semiconductor. The experiments presented here demonstrate the potential of this technique for rapidly determining degenerate nonlinear absorption spectra by, for example, replacing the detectors and narrowband filters with a spectrometer and CCD array. This avenue is currently being pursued. In addition, we are extending this "open aperture" Z-scan method with the "closed aperture" [13] $\mathrm{Z}$-scan to determine the degenerate nonlinear refraction (i.e. $\mathrm{n}_{2}$ ) spectrum as well.

We expect that with larger temporal dispersion (e.g. by using a thicker $\mathrm{ZnSe}$ slab for dispersion) spatial separation may not be needed. This would require higher spectral energy densities for the WLC but fortunately this possibility has already been demonstrated with $\mathrm{BaF}_{2}[9]$.

The method presented here combined with the nondegenerate measurements obtained by using our WLC femtosecond pump-probe method [6] should yield a very complete picture of the nonlinear properties of a material, allowing for the full characterization of their absorptive nonlinearities and the associated dispersion of the nonlinear refraction. The results discussed in this article were presented at CLEO 2004 [14]. 